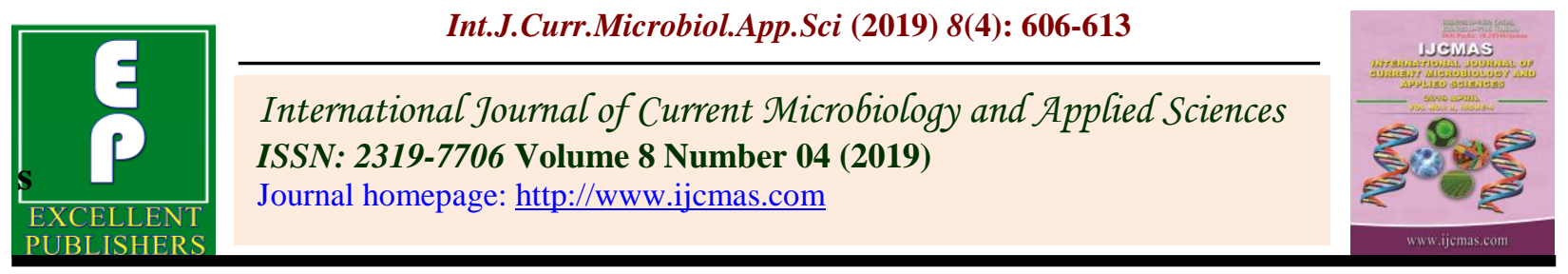

Original Research Article

https://doi.org/10.20546/ijcmas.2019.804.066

\title{
Study of Bacterial and Mycological profile of Cases of Ear Infections in Tertiary Care Hospital in Bhavnagar, Gujarat
}

\author{
Ankita Nisarta and Rakesh Rajat* \\ Department of Microbiology, GMERS Medical College, Himmatnagar, Gujarat, India \\ *Corresponding author
}

A B S T R A C T

\begin{tabular}{|l|}
\hline K e y w o r d s \\
Ear Infection, \\
Pseudomonas \\
aeruginosa, \\
Antibiotic \\
sensitivity test \\
\hline Article Info \\
\hline Accepted: \\
07 March 2019 \\
Available Online: \\
10 April 2019 \\
\hline
\end{tabular}

Ear infections usually results from bacterial, fungal, viral infections. Ear infections are major health problem and occur with a high incidence and prevalence in both developed and developing countries which leads to deafness and intra-cranial complications. It may be acute, chronic or recurrent. It can be suppurative or non-suppurative. The Aim of study is to determine Bacterial and Mycological profile and their antimicrobial sensitivity pattern of Ear Infections. Total 95 patients of Ear Infections are included in this study. Swabs were taken and sent immediately to the microbiology laboratory and processed. Bacterial and Fungal isolates were identified using standard methods and antibiotic susceptibility testing was done. Pseudomonas aeruginosa 21 (44\%) was the most predominant bacterial isolate followed by Staphylococcus aureus 16 (34\%). Among fungi, Aspergillus niger 10 (16\%) was common isolate, followed by Candida albicans 2 (3\%). The study of microbial pattern and their antibiotic sensitivity determines the prevalent bacterial organisms causing Ear Infections in local area and to start its empirical treatment and complications for successful outcome, thus to prevent the emergence of resistant strains.

\section{Introduction}

The ear is the organ responsible for hearing and also maintaining balance, it's divided into the outer, middle and inner ear with the outer and middle regions being most susceptible to injury and infections.(1) Ear is an important sensory organ. It is worthy to note that ear infections are a very common problem worldwide. Ear infection is an inflammation of the ear and ear discharge is one of the commonest symptoms of ear infection. Ear discharge may arise from external auditory meatus in otitis externa or middle ear cavity in otitis media.(2) Ear infections are common in babies, young children, especially those aged six to 18 months and adults. Most children will have an ear infection before the age of five. $(1,6)$ but the magnitude is different in different countries $(2,6)$ about 65-330 million people suffer from ear infection worldwide and $60 \%$ of them had significant hearing impairment. $(2,6,12)$ Microbial agents can infect the middle and external parts of the ear and may involve the skin, cartilage, periosteum, ear canal, and tympanic and mastoid cavities. Ear infection can be classified as acute suppurative otitis media (ASOM), chronic suppurative otitis media (CSOM), or otitis externa $(\mathrm{OE})(4,8)$. 
Chronic Suppurative Otitis Media (CSOM) is a chronic inflammation of middle ear due to various causes. It is famous for its recurrence and persistant infection. It is one of the common causes of deafness and can also cause permanent perforation $(3,7)$. It is one of the most common chronic diseases of childhood. Its chronic form is a serious problem in all age groups with less chance of recovery. (4) It is one of the major causes of deafness in India. $(2,7)$ A leading cause of hearing loss in younger ages, particularly in low and middle-income countries, is untreated ear infections, often with discharge from the ear. Vaccine-preventable infectious diseases such as rubella, meningitis, measles, or mumps can also lead to hearing loss (4).

Anatomically the children's Eustachian tube is shorter, more horizontal with a more flaccid cartilage which can easily impair its opening and hence ear infection is a major health problem of them especially in those with poor socioeconomic status. $(2,10)$ CSOM occurs due to various reasons. The disease usually occurs after upper respiratory viral infections followed by invasion of pyogenic organisms. $(3,9)$

CSOM can cause severe adverse effects like intra and extra cranial complications which can be life threatening. $(3,9)$ In certain cases this condition can lead to serious lifethreatening complications, such as hearing impairment, brain abscesses, or meningitis, mostly in childhood and late in life $(4,11)$. The incidence of CSOM is increasing in the developing countries because of the poor hygienic practices and lack of health education. The complications of CSOM have been reduced to a greater extent because of the invention of antibiotics. But irrational use of antibiotics has lead to the emergence of resistant organisms to the commonly used drugs $(3,9)$.
The causative agents of ear infection might be bacterial, viral, or fungal. $(4,10)$ Commonly found pathogens in CSOM are Pseudomonas aeruginosa, Staphylococcus aureus, Proteus mirabilis, Klebsiella pneumoniae, Escherichia coli, Aspergillus spp and Candida spp (5). Moreover, antimicrobial resistance profile of bacteria varies among population because of difference in geography, local antimicrobial prescribing practices and prevalence of resistant bacterial strains. (12)

To Study Bacterial and Fungal Etiology and antimicrobial sensitivity pattern of Ear Infections in Sir Takhatsinhji General Hospital, Bhavnagar.

\section{Materials and Methods}

Study period was from December 2012 to December 2013.Total 95 Patients with ear infections were included in the study. All samples were processed following standard bacteriological procedures. Three samples were collected in sterile swabs, one for direct Gram's stain \& $\mathrm{KOH}$ mount, second for bacterial culture \& third for fungal culture.Pus samples were inoculated on to blood agar, chocolate agar, Mac conkey's agar and Sabouraud dextrose agar. The plates were incubated overnight at $37^{\circ} \mathrm{C}$.

The growth of organism was identified by characteristic colony morphology and appropriate biochemical reactions, direct microscopy with $\mathrm{KOH}$ preparation \& fungus culture. The Antibiotic Susceptibility Pattern of bacterial isolates was done by Kirby Bauer disc diffusion method as per CLSI (Central Laboratory Standard Institute) guidelines. The antibiotic discs and their potency (antibiotic content) that was used for susceptibility testing of bacterial isolates are listed below: 


\begin{tabular}{|l|l|}
\hline Anitbiotic discs & Potency \\
\hline Ampicillin & $10 \mathrm{ug}$. \\
\hline Penicillin $-\mathrm{G}$ & $10 \mathrm{units}$ \\
\hline Linezolid & $30 \mathrm{ug}$. \\
\hline Vancomycin & $30 \mathrm{ug}$ \\
\hline Gentamicin & $30 \mathrm{ug}$ \\
\hline Ciprofloxacin & $5 \mathrm{ug}$. \\
\hline Tetracycline & $30 \mathrm{ug}$. \\
\hline
\end{tabular}

\section{Results and Discussion}

Out of 95 samples from patients enrolled in the study, 63(66\%) Samples were culture positive. 32 samples show no growth of organism.

In our study group, maximum numbers of cases were from the male group 51(54\%) cases and females were 44(46\%). The occurrence of gram negative organisms (GNB) was higher than for gram positive organisms (GPC) with GNB 35/63 (56\%), GPC 16/63 (25\%) and fungi 12/63 (19\%). The Most common organism isolates were Pseudomonas aeruginosa 21 (44\%) and Staphylococcal aureus 16(34\%), followed by Klebsiella 08 (17\%), E.coli 4 (8\%), Proteus mirabilis 2 (4\%). Among fungi, Aspergillus niger $10(16 \%)$ was common isolate, followed by Candida albicans $2(3 \%)$.

In present study (Table 3) showed GNB were most effective to imipenem and meropenem $100 \%$, tetracycline $80 \%$ and GPC were most effective to vancomycin $100 \%$, linezolid $84 \%$ and tetracycline 96\% (Table 1-4 and Fig. 15).

Table.1 Number of isolates

\begin{tabular}{|l|l|}
\hline Positive growth & $\mathbf{6 3}(66 \%)$ \\
\hline No growth & $32(34 \%)$ \\
\hline Total samples & 95 \\
\hline
\end{tabular}

Table.2 Type of Isolates

\begin{tabular}{|l|l|}
\hline Organism & No of Isolates \\
\hline Bacterial & $51(81 \%)$ \\
\hline Fungal & $12(19 \%)$ \\
\hline
\end{tabular}

Table.3 Antibiogram of gram-negative isolates

\begin{tabular}{|c|c|c|c|c|c|c|c|c|c|c|c|c|}
\hline Orgaisms & $\begin{array}{l}\text { AMP } \\
\%\end{array}$ & $\begin{array}{l}\text { AK } \\
\%\end{array}$ & $\begin{array}{l}\text { GE } \\
\%\end{array}$ & $\begin{array}{l}\text { AS } \\
\%\end{array}$ & $\begin{array}{l}\text { CTX } \\
\%\end{array}$ & $\begin{array}{l}\text { CAZ } \\
\%\end{array}$ & $\begin{array}{l}\text { CIP } \\
\%\end{array}$ & $\begin{array}{l}\mathbf{C X} \\
\%\end{array}$ & $\begin{array}{l}\text { TE } \\
\%\end{array}$ & $\begin{array}{l}\text { PIT } \\
\%\end{array}$ & $\begin{array}{l}\text { IPM } \\
\%\end{array}$ & $\begin{array}{l}\text { MRP } \\
\%\end{array}$ \\
\hline $\begin{array}{l}\text { Pseudomonas } \\
\text { aeruginosa }\end{array}$ & 42 & 72 & 73 & 56 & 75 & 68 & 70 & 64 & 82 & 65 & 98 & 98 \\
\hline Klebsiella & 43 & 68 & 75 & 61 & 63 & 55 & 75 & 50 & 80 & 60 & 100 & 100 \\
\hline E.coli & 52 & 74 & 81 & 74 & 70 & 75 & 80 & 65 & 75 & 72 & 100 & 100 \\
\hline $\begin{array}{l}\text { Proteus } \\
\text { Mirabilis }\end{array}$ & 44 & 60 & 68 & 52 & 64 & 60 & 72 & 58 & 76 & 54 & 96 & 96 \\
\hline
\end{tabular}


Int.J.Curr.Microbiol.App.Sci (2019) 8(4): 606-613

Table.4 Antibiogram of gram-positive isolates

\begin{tabular}{|l|l|l|l|l|l|l|l|l|l|l|l|l|}
\hline Orgaisms & AK & GE & TE & CIP & CH & COT & CX & E & OX & LZ & VA & P \\
\hline $\begin{array}{l}\text { Staphylococus } \\
\text { aureus }\end{array}$ & $76 \%$ & $70 \%$ & $96 \%$ & $65 \%$ & $70 \%$ & $72 \%$ & $55 \%$ & $68 \%$ & $47 \%$ & $84 \%$ & $100 \%$ & $37 \%$ \\
\hline
\end{tabular}

Fig.1 GRAM stain of GNB in microscopic view
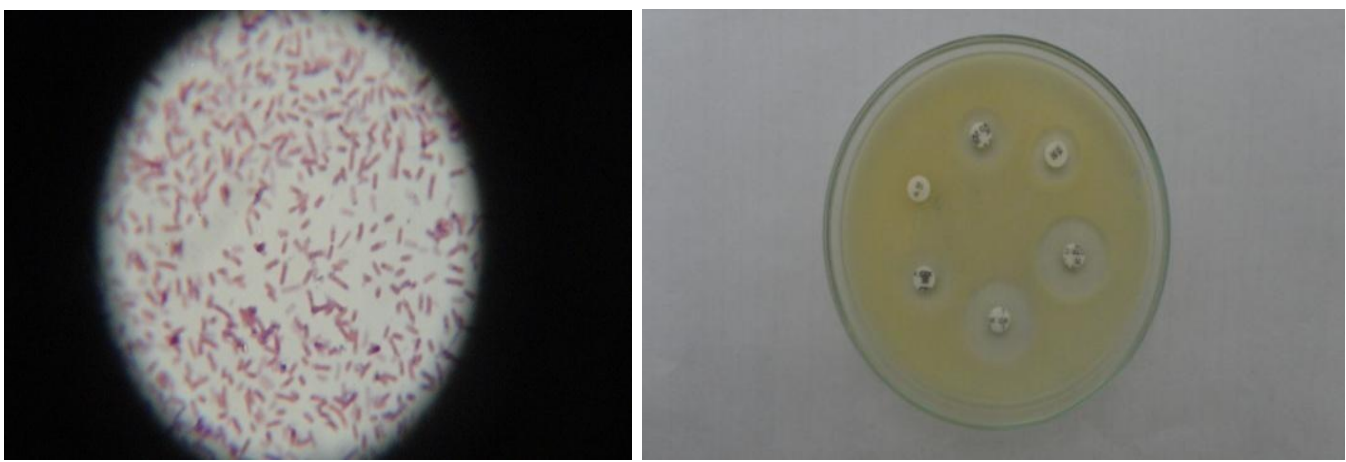

Fig.2 Gram stain of GPC in microscopic view

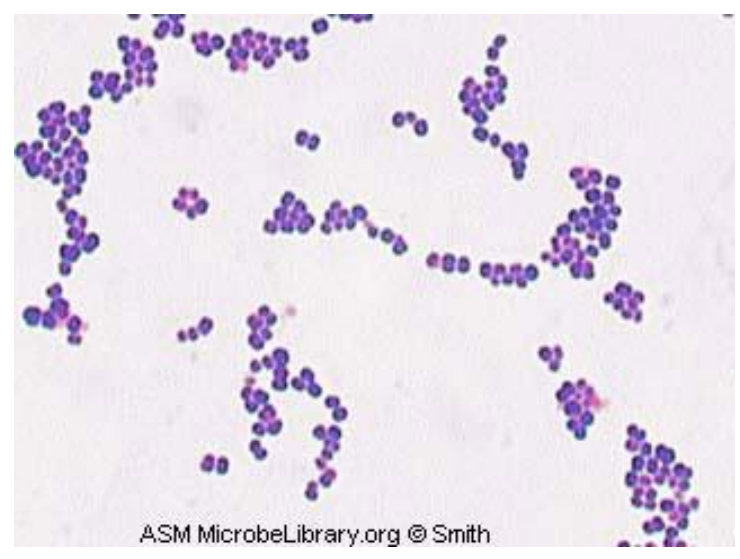

Fig.3 Aspergillus niger growing on Sabouraud's Dextrose Agar

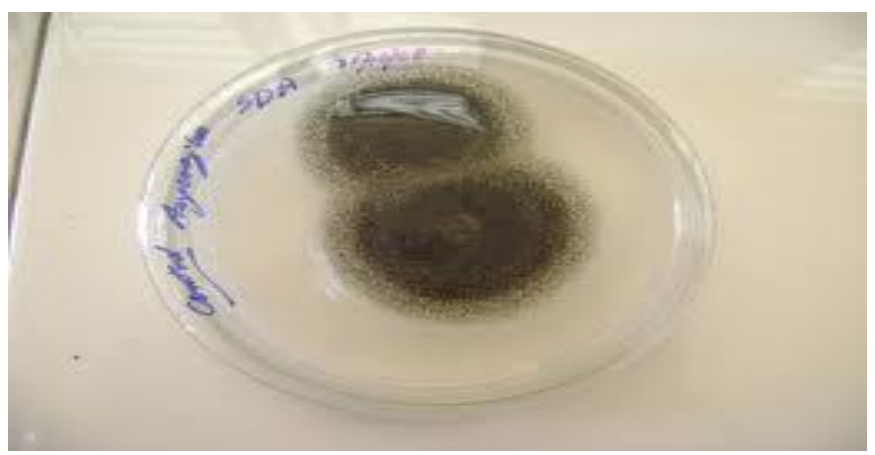


Fig.4 Sex wise distribution of cases

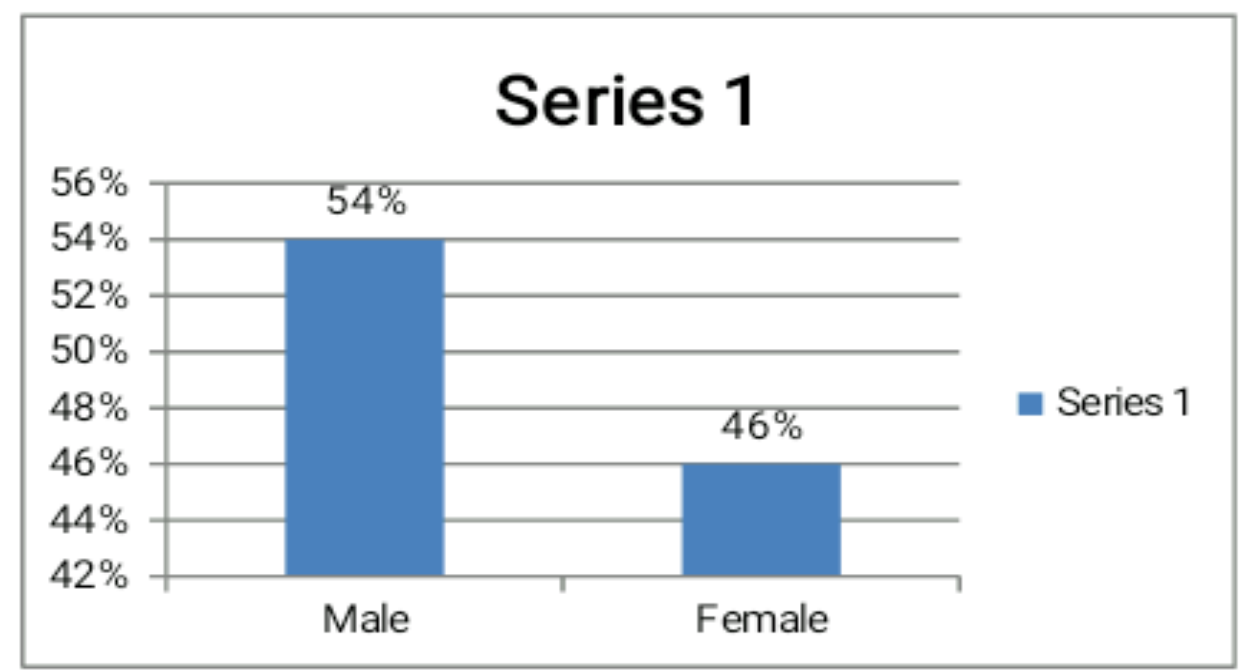

Fig.5 Bacterial and fungal agents isolated from ear infection

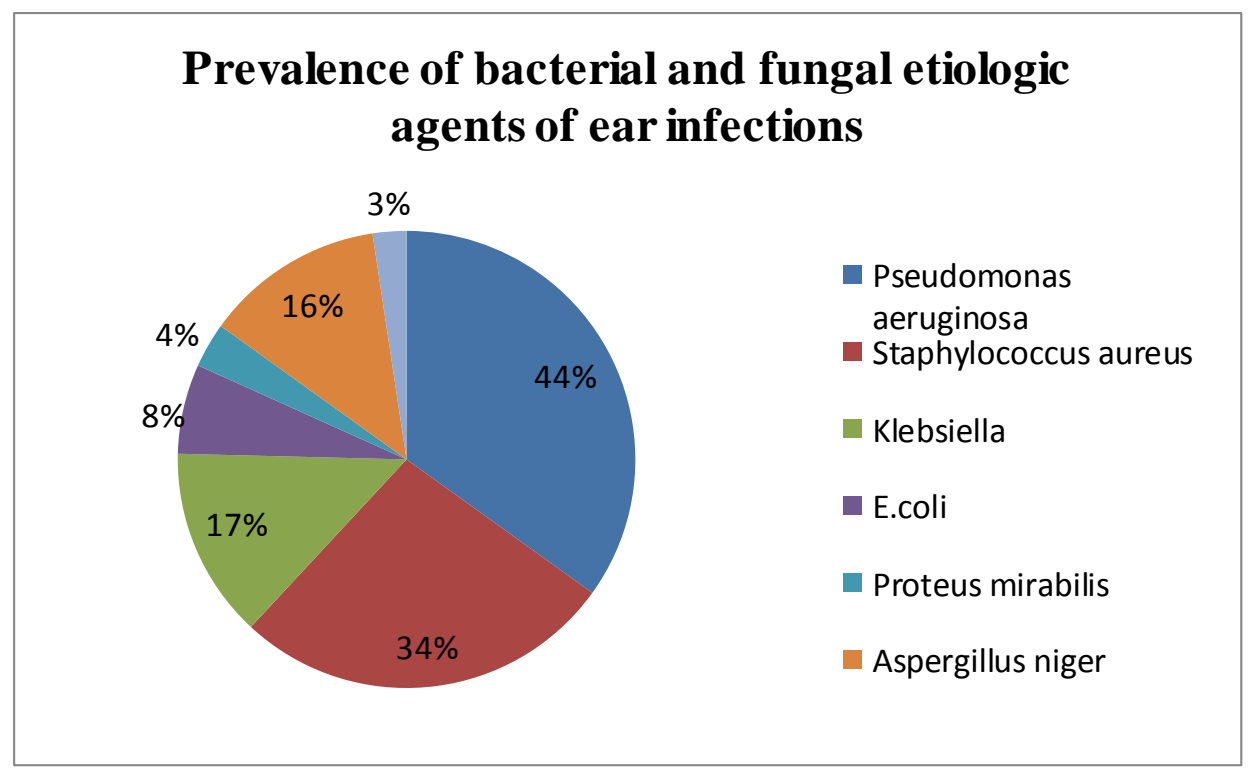

In Present study, Out of total 95 specimens, 32 shows no growth of organism, while 63 specimens shows growth of one or two organisms. Such negative cultures may have been result of the modification of bacterial flora in the affected ears by prior empirical antibiotic therapy (10)

In our study, males (51) were more commonly affected than females (44) and which is in concordance with findings of
Rakeshkumar et al., (2013) (7) in which males were $83 \%$ and females were $61.73 \% .(7)$

In the present study, the age of the patients ranged from $1 \mathrm{yr}$ to $80 \mathrm{yrs}$. Maximum number of cases were found in the age group of 1 - 10 yrs, followed by $11-20$ yrs. This could be due to the short, wide and straight Eustachian tube, lower immunity of children compared to adults and the fact that bacteria adhere better 
to epithelial cells of children that adults in infants and children $(11,15)$. So, they are more prone to upper respiratory tract infections (7).

Pseudomonas species was the most commonly isolated organism in our study. It is a common environmental organism usually found in warm and moist environment, and is known to colonize the external auditory canal. It is commonly associated with otitis externa and chronic superlative otitis media. (8) They are known for the ability to resist to antibiotics. Moreover, $P$. aeruginosa uses its pili to attach to the necrotic or diseased epithelium of the middle ear. Once attached, the organism produces enzymes like proteases to elude the normal defense mechanism of body required for fighting infections.(6) The prevalence of $K$. pneumoniae and $E$. coli in this study was $17 \%$ and $8 \%$, respectively. Isolation of fecal bacteria like K. pneumoniae and $E$. coli might indicate that individuals were at risk of infection due to poor hygiene conditions (6).

In the present study, the most common bacterial isolates were Pseudomonas aeruginosa (44\%), Staphylococcus aureus (34\%), Klebsiella (17\%), Escherichia coli (8\%), Proteus species (4\%), which is similar to studies performed by Rakesh Kumar et al., (7) and L. APPIAH-KORANG et al., (8). But in contrast Arti Agrawal et al., reported Staphylococcus aureus as the major causative agent (9). The slight differences observed in the isolates and species may be because of geographical and/or ethnic variations. Our finding is in randem with the pattern of CSOM infection within the tropical region. It is seen that both gram positive and gram negative organisms are responsible for infection of middle ear (7)

Among fungi, Aspergillus niger 10 (16\%) was common isolate, followed by Candida albicans $2(3 \%)$. Since fungus can thrive well in moist pus, so, fungal infections of the middle-ear are common (13) Aspergillus niger is an opportunistic filamentous fungi, it has been identified as the cause of bilateral otomycosis. Aspergillus niger grows on cerumen, epithelial scales and detritus deep in the external canal. The resulting accumulation of these inflammatory materials along with cerumen and fungal debris result in plug formation, which is extremely significant and usually leads to diminished hearing ability; pruritis, irritation of the surface layer of the external ear itself is a predisposing factor for bacterial colonization. There may be superficial erosion of membranes (15)

All the isolates in present series were tested against various antibiotics. (14) Pseudomonas showed high sensitivity to ciprofloxacin $(92.3 \%)$, gentamicin $(84.61 \%)$, imipenem $(84.61 \%)$, piperacillin $(88.46 \%)$. High fluoroquinolones antibacterial activity against Pseudomonas isolates was reported by others, although resistant strains of Pseudomonas isolates to fluoroquinolones were detected in other studies (2).

Staphylococcus aureus was sensitive to gentamicin $(90.47 \%)$, ciprofloxacin $(90.47 \%)$, clindamycin $(85.7 \%)$, cephalexin $(85.7 \%)$ and ofloxacin $(71.42 \%)$. Clinical resistance of Staphylococci spp. to penicillin and other antimicrobial agents is now a problem throughout the world. Staphylococci spp. sensitivity to ciprofloxacin is in agreement with other reports and most of the investigators reported high sensitivity rate for Staphylococci spp. to fluoroquinolones such as ofloxacin and ciprofloxacin (2). The therapeutic use of antibiotics is usually started empirically prior to results of microbiological culture. Selection of any antibiotic is influenced by its efficacy, resistance of bacteria, safety, risk of toxicity and cost. Knowledge of the local microorganism 
pattern and their antibiotic sensitivity is then essential to allow for effective and costsaving treatment. Microbiology cultures yield multiple organisms and these vary depending on climate, patient population and previous antibiotics have or have not been recently used (13)

Antibiotic susceptibility patterns serve as a useful guideline for choosing the appropriate antibiotic (11). In the era of antibiotics, the emergence of antibiotic resistance is becoming more common. Patient non compliance is an important factor responsible for the development of antibiotic resistance (15)

In this study, Pseudomonas aeruginosa was most common isolate followed by Staphylococcal aureus. Knowledge of the pathogens \& antibiotic sensitivity pattern responsible for Ear infections \& choosing suitable antibiotics according to susceptibility tests should guide the management of disease treatment \& reduce the burden of infection on the patients \& in the long term it may reduce the cost of treatment. Early microbiological diagnosis of CSOM ensures prompt and effective treatment to avoid complications.

\section{References}

1. Dilshad Arif, Rakesh Kumar Mukhia, Sanjeeva Kumar Goud et al, Bacteriological profile of ear infections and its antibiotic susceptibility pattern in tertiary care hospital Navi Mumbai, Journal Of Dental And Medical Sciences (IOSR-JDMS), Volume 13, Issue 5, (May. 2014), PP 58-62.

2. Raakhee T, Sreenivasa Rao Unguturu et al., Bacteriological Study Of Discharging Ear In Patients Attending A Tertiary Care Hospital, International Journal Of Research In Medical Sciences, 2014 May; 2(2): 602-606
3. Prakash M, Lakshmi K, Anuradha S1, Swathi Gn et al., bacteriological profile and their antibiotic susceptibility pattern of cases of chronic suppurative otitis media Asia Journal of Pharmaceutical and Clinical Research, Vol 6, Suppl 3, 2013

4. Ayele Argaw-Denboba, 1 Asrat Agalu Abejew et al., Antibiotic-Resistant Bacteria Are Major Threats Ofotitis Media In Wollo Area, Northeastern Ethiopia: A Ten-Year Retrospective Analysis, International Journal Of Microbiology volume 2016, Article ID 8724671, 9 Pp.

5. Ghulam Fatima, Maria Shoaib et al., Antimicrobial susceptibility pattern of bacterial and fungal isolates from patients with chronic suppurative otitis media in perspective of emerging resistance Pakistan Journal of Otolaryngology 2013; 29: 49-53.

6. Derese Hailu, Daniel Mekonnen, Awoke Derbie et al., Pathogenic bacteria profile and antimicrobial susceptibility patterns of ear infection at Bahir Dar Regional Health Research laboratory Center, Ethiopia, Hailu et al. Springer Pus (2016) 5:466DOI 10.1186/S40064-0162123-7

7. Rakesh Kumar, P Srivastava et al., isolation and antimicrobial sensitivity profile of bacterial agents in chronic suppurative otitis media patients at Nims Hospital, Jaipur, IJPBS, 3(4): 2013, 265 269

8. L. Appiah-Korang1, S. Asare-Gyasilet $a l$., aetiological agents of ear discharge: a two year re-view in a teaching hospital In Ghana, Ghana Medical Journal, June 2014 Volume 48, Number 2.

9. Arti Agrawal, Dharmendra Kumar1, Ankur Goyal et al., Microbiological profile and their antimicrobial sensitivity pattern in patients of otitis media with ear discharge, Indian Journal Of 
Otology, January 2013, Vol 19, Issue 1

10. Sunilkumar Biradar And C. Roopa et al, Study of microbiological profile and their antibiogram in patients with chronic suppurative otitis media, International Journal of Current Microbiology and Applied Sciences, Volume 4 Number 9 (2015) Pp. 981-985

11. Y. K. Harshika, S. Sangeetha And R. Prakash et al, Microbiological Profile Of CSOM And Their Antibiotic Sensitivity Pattern In A Tertiary Care Hospital, Int.J.Curr.Microbiol.App.Sci (2015) 4(12): 735-743

12. B.L. Chaudhary, Snehanshu Shukla et al, Bacteriological profile and their antibiotic susceptibility pattern in cases of otitis media, Bopams, Vol.2. Issue. 2.;
2014

13. Asima Banu1, B. Viswanath et al, Microbiological study and drug susceptibility of organisms causing cholesteatoma, Jermm, 2015; Vol. 1, Issue 1, July-December 2015.

14. Sateesh Kumar Malkappa et al, Study of aerobic bacterial isolates and their antibiotic susceptibility pattern in chronic suppurative otitis media. Indian J Otol 2012, 18(3): 136-139.

15. Favour Osazuwa et al, Etiologic agents of otitis media in Benin city, Nigeria, najms. 2011. 395.

16. Mackie \& McCartney, Practical Medical Microbiology, $14^{\text {th }}$ Edition, Page no-6061.

\section{How to cite this article:}

Ankita Nisarta and Rakesh Rajat. 2019. Study of Bacterial and Mycological profile of Cases of Ear Infections in Tertiary Care Hospital in Bhavnagar, Gujarat. Int.J.Curr.Microbiol.App.Sci. 8(04): 606-613. doi: https://doi.org/10.20546/ijcmas.2019.804.066 